\title{
Numerical Simulation of Non-Destructive Characterization of CFRP Composite Through Frequency Modulated Thermal wave Imaging
}

\author{
T. S. V. Saketh, V. V. N. S. G. Sathwik, D. V. Sai Prahlad, V. Gopi Tilak, G. V. P. Chandra Sekhar \\ Yadav, V. S. Ghali
}

\begin{abstract}
Non-destructive testing plays a vital role in industrial and biomedical applications. Non-stationary stimulation based active Infrared thermography is an emerging area of interest in subsurface defect detection and visualization. In present article, frequency modulated thermal wave imaging is employed on a numerical simulation to detect defects of CFRP specimen and applied various post processing techniques such as FFT phase, Pulse compression, principal component analysis and random projection transform for better defect detection. Defect Signal to noise ratios considered as merit of analysis.

Keywords: Frequency modulated thermal wave imaging, Signal to noise ratio, Non-stationary thermal wave imaging, CFRP, Spectral reshaping, Pulse compression, PCA, Random projection transform.
\end{abstract}

\section{INTRODUCTION}

Ever since the non-stationary thermal wave imaging (NSTWI) technique proposed, they became an active area of interest over thermal non-destructive testing modalities due to their depth scanning capabilities [1]. In NSTWI, the moderate peak power optical stimulus is modulated by a band of low frequencies such that the projected energy produces diffusive heat wave that diffuse into the object into deeper depths. The reflected thermal wave from the subsurface layer further creates a thermal contrast over the surface of the object is post

Manuscript published on November 30, 2019.

* Correspondence Author

T. S. V. Saketh*, B.Tech, Department of Electronics and Communication Engineering, Koneru Lakshmaiah Education Foundation, Vaddeswaram, AP, India.

V. V. N. S. G. Sathwik, B.Tech, Department of Electronics and Communication Engineering, Koneru Lakshmaiah Education Foundation, Vaddeswaram, AP, India.

D. V. Sai Prahlad, B.Tech, Department of Electronics and Communication Engineering, Koneru Lakshmaiah Education Foundation, Vaddeswaram, AP, India.

V. Gopi Tilak, PhD Scholar, Department of Electronics and Communication Engineering, Koneru Lakshmaiah Education Foundation, Vaddeswaram, AP, India.

G. V. P. Chandra Sekhar Yadav PhD Scholar, Department of Electronics and Communication Engineering, Koneru Lakshmaiah Education Foundation, Vaddeswaram, AP, India.

V. S. Ghali*, Professor, Department of Electronics and Communication Engineering, Koneru Lakshmaiah Education Foundation, Vaddeswaram, AP, India.

(C) The Authors. Published by Blue Eyes Intelligence Engineering and Sciences Publication (BEIESP). This is an open access article under the CC-BY-NC-ND license http://creativecommons.org/licenses/by-nc-nd/4.0/ processed for in-detail defect visualization. The non-stationary stimulus can be produced by methods like linear frequency modulation [2], its digitized version [3] or quadratic frequency modulation [4]. A binary phase modulation scheme also introduced with uses 7 bit barker coded stimulus [5]. Though the conventional mechanisms like pulse thermography (PT) [6-8] and Lock-in thermography (LIT) [9] established in various industries, NSTWI techniques grab interest due to the capabilities of projecting more energies into object with moderate peak power stimulus into deeper depths due to band of frequencies.

On the acquired thermal response, a first order polynomial fit is employed to fit the trend and retrieve the dynamic component by subtracting mean from each thermal response. Further various post processing schemes have been employed for further analysis of subsurface anomaly [10-20]. General post processing scheme employed over various stimulation mechanisms is Fourier transform based phase analysis, since phase is more immune to non-uniform radiation emissions of the object. Fourier transform result in a phase response with showing maximum thermal contrast between defects and non-defective region at a certain frequency. Since the stimulation is a non-stationary chirp, the acquired thermal response is also a non-stationary chirp and Fourier transform outperform for non-stationary signals to extract sub-harmonics of low frequency chirps which are key factors for deeper defects identification [5, 6]. Various processing schemes have been introduced to overcome the limitation associated with Fourier transform. A time domain approach named Pulse compression, in which thermal response is cross correlated with a pre-selected non-defective thermal profile and corresponding peak time delays analyzed for defect depth quantification [10]. Similarly, the correlation employed in frequency domain (convolution) by Hilbert transforming the reference signal and analyzing obtained phase response known as Hilbert phase approach results in time domain phase response [11]. Further, a theoretical model is provided for defect depth quantification based on chirp $\mathrm{Z}$ transform approach for NSTWI [12]. Time frequency analysis based methods such as wavelets [13], empirical mode decomposition [14] further improve the defect depth quantification capabilities, presented in [15]. 


\section{Numerical Simulation of Non-Destructive Characterization of CFRP Composite Through Frequency Modulated Thermal wave Imaging}

Similarly, Statistical approaches like principal component analysis [16], Independent

$\mathrm{s}$ the detection and defect classification criteria's for NSTWI $[23,24]$. Further, in detail understanding of observed defects with their respective locations is presented by fusing the thermograms onto the original sample optical image [25].In present article, various post processing schemes have been analyzed for defect detection capability of FMTWI system for a CFRP with flat bottom holes of different sizes at different depths. Applied schemes are FFT phase, pulse compression; principal component analysis and random projection transform techniques. Defect Signal to noise ratios are considered for analyzing these techniques as figure of merit and observations provide that random projection transform performs good in defect visualization with good SNRs.The article organized as follows, a brief introduction to FMTWI; along with different post processing techniques is presented in section II. Section III deals with the experimentation and methodology of post processing, section IV gives an in detail discussion over obtained results and finally the article concluded in section V.

\section{THOERY}

\section{A. FMTWI}

The schematic of FMTWI is shown in fig. 1.a, in which a linear frequency modulated optical heat flux of moderate peak power from a set of halogen lamps is imposed on the front surface of the object. The linear frequency excitation is given by

$$
\left.\frac{\partial T}{\partial t}\right|_{x=0}=Q_{0} e^{-j\left(a t+b t^{2}\right)}
$$

Where, $Q_{0}$ is intensity of heat flux, $a$ is initial frequency and $b$ is bandwidth of the signal. This excitation creates a thermal in-equilibrium over the object surface, which generates diffusive heat waves to propagate into subsurface layers of the object. The 1 dimensional heat diffusion is given by a homogeneous differential equation as

$$
\frac{\partial^{2} T}{\partial x^{2}}=\frac{1}{\alpha} \frac{\partial T}{\partial t}
$$

Solving eqn. 2 under boundary conditions and applied stimulus results in temperature over the surface as [5]

$$
\begin{aligned}
& T(x, \mathrm{t})=Q_{0} e^{-x / \mu}[\cos (k)+j \sin (k)] \\
& k=2 \pi\left(a t+\frac{b t^{2}}{2 T}\right)-x / \mu
\end{aligned}
$$

\section{B. POSTPROCESSING TECHNIQUES}

\section{- FFT PHASE}

In FFT phase, each thermal response is processed by a fast Fourier transform which results in complex temperature values in at discrete frequency instants. Taking phase value from these complex values results in phasegrams with better thermal contrast whereas magnitude response is more robust to non-uniformities in the thermograms. The application of Fourier transform is given by

$$
\begin{aligned}
& F(\omega)=F F T(f(n))=\sum_{n=0}^{N-1} f(n) e^{j \omega n} \\
& =\sum_{n=0}^{N-1} \operatorname{Re}(F(n \omega))+\operatorname{Im}(F(n \omega))
\end{aligned}
$$

The corresponding phase at any frequency from real (Re) and imaginary (Im) components can be given by

$$
\phi(n)=\tan ^{-1}\left(\frac{\operatorname{Im}(F(n \omega))}{\operatorname{Re}(F(n \omega))}\right)
$$

\section{- PULSE COMPRESSION}

Pulse compression is a Cross correlation based time domain approaches in which the correlated thermal response possess peak at the center of sequence with varying peak delay correspond to defective and non-defective counterpart. The cross correlation is employed over each thermal response $\mathrm{g}(\mathrm{t})$ with a preselected thermal response $\mathrm{h}(\mathrm{t})$ from non-defective region. These peak delays are proportional to dime delays of reflected thermal responses from defects at different depths. The cross correlation of thermal response is mathematically represented by [10]

$$
R(t)=\int_{-\infty}^{\infty} h(t) g(t+\tau) d t
$$

\section{- PRINCIPAL COMPONENT ANALYSIS}

PCA is an optimized representation of a signal or data sequence with a linearly dependent orthogonal basis vectors of reduced dimension. These basis vectors for thermographic data are computed from Eigen decomposition of covariance matrix. Based on the Eigen values distribution, projection of few of these orthogonal basis vectors into either data driven or pre computed coefficients produces principal components of reduced dimension. This PCA and following random projection transform needs the $3 \mathrm{D}$ thermographic data to be reshaped into $2 \mathrm{D}$ before processing and at the end, the obtained principal components of random projection components are again reshaped into 3D for thermographic representation. The projection of these Eigen vectors V into the original data $\mathrm{S}$ is given by [9]

$$
P C S=\sum_{n=1}^{L} V^{T} S \text { where } L<<N
$$

\section{- RANDOM PROJECTION TRANSFORM}

As mentioned earlier, the initial 3D thermal response is converted into a $2 \mathrm{D}$ matrix by rearranging thermograms into columns of the matrix. Then Gram-Schmidt algorithm is used to generate a random matrix composed of orthonormal basis vectors. Further the data driven thermal response is projected onto these random matrix and corresponding coefficients are rearranged to form random projection sequence of thermograms [18]. 


\section{EXPERIMENTATION}

A $16 \mathrm{cmX} 16 \mathrm{cmX} 0.5 \mathrm{~cm}$ CFRP composite back holes at different depths having different diameters designed and simulated with LFM heat flux at front plane in COMSOL Multiphysics software.

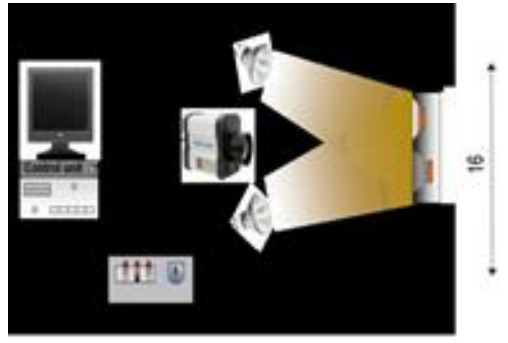

a

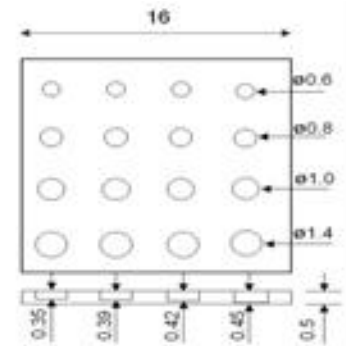

b
Fig 1 a. Experimental schematic of QFMTWI and $b$. Layout of CFRP sample (all dimensions in mm).

The sample layout is shown in fig 1 . b. A $2 \mathrm{KW}$ excitation modulated by a frequency sweep starting from $0.01 \mathrm{~Hz}$ to $0.1 \mathrm{~Hz}$ is imposed for 100 seconds. Respective temporal thermal map acquired at a rate of 25 frames per sec. This captured thermal response has been processed with various post processing techniques employed to extract the subsurface details.

\section{RESULTS AND DISCUSSION}

On the acquired thermal response, FFT phase, Pulse compression, principal component analysis and Random projection transform techniques are employed. Corresponding results shown in fig. 2.a, b, c and d for FFT phase at $0.027 \mathrm{~Hz}$, Pulse compression at 16.08sec, 2nd PCA and 1st random projection component respectively. It is observed from the results that random projection transform provides better results in defect visualization compared to other processing schemes.
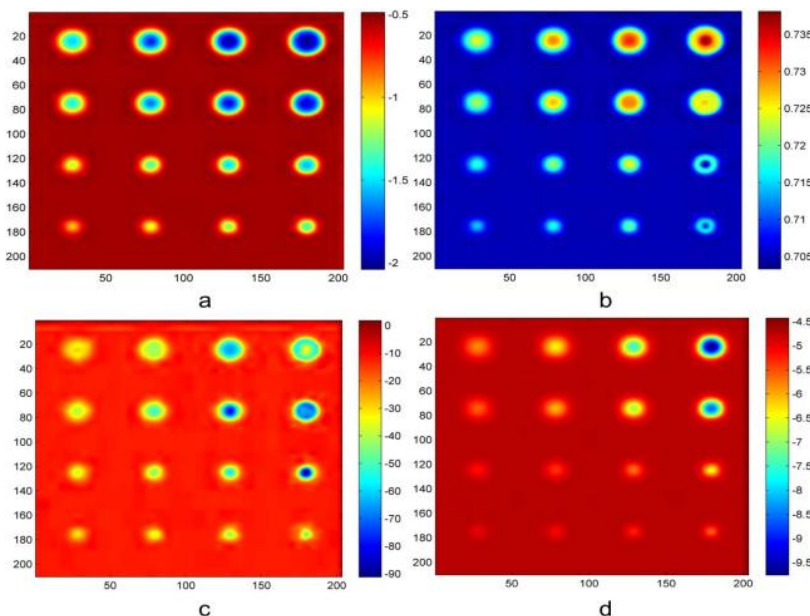

Fig 2 a. FFT phase at $0.027 \mathrm{~Hz}$, b. Pulse compression at $16.08 \mathrm{sec}, \mathrm{c}$. 2nd principal component and 1st random $d$. projection component for raw thermal response.

Further signal to noise ratio is employed on each defects for afore mentioned processing techniques. SNR is the ratio between differences in means of defective region to non-defection region to the std of non-defective region. Fig. 3 depicts the SNR values,, inferring that random projection transform gives better signal to noise ratios.

$$
S N R(d B)=20 \log \left(\frac{\mu_{\text {Defective }}-\mu_{\text {Non-Defective }}}{\sigma_{\text {Non-defective }}}\right)
$$

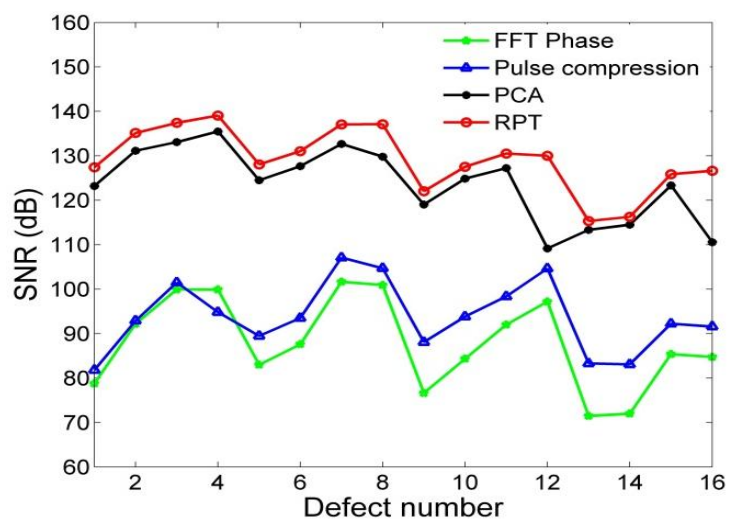

Fig 3. Signal to noise ratios of different post processing techniques employed on CFRP sample

\section{CONCLUSION}

In present article, a CFRP sample with back holes is examined by imposing LFM heat flux on the front plane through numerical simulation. On the observed thermal response, various post processing schemes have been employed and from the observed thermograms, a conclusion is given that random projection transform gives better signal to noise ratios supporting its detection capability.

\section{REFERENCES}

1. R Mulaveesala, V S Ghali and V Arora, "Applications of non-stationary thermal wave imaging methods for characterization of fiber-reinforced plastic materials," Electronic letters, 49(2), pp-16-18 (2013).

2. Ravibabu Mulaveesala and Suneet Tuli, "Theory of frequency modulated thermal wave imaging for non-destructive subsurface defect detection", Applied Physics Letters 89, 191913-2006.

3. Vanita Arora, Ravibabu Mulaveesala, Anju Rani \& Anshul Sharma, "Digitised Frequency Modulated Thermal Wave Imaging for Non-destructive Testing and Evaluation of Glass Fibre Reinforced Polymers", Nondestructive Testing And Evaluation, 13 Nov 2018.

4. G V. Subbarao and R. Mulaveesala, "Quadratic frequency modulated thermal wave imaging for Non- destructive testing", Progress in Electromagnetics Research M, Vol. 26, 11-22, 2012.

5. V.S.Ghali, P.S.S.Balaji and R.Mulaveesala, "Barker coded thermal wave imaging for defect detection in carbon fibre-reinforced plastics,” Insight, 53, pp.621-624 (2011).

6. Maldague X P V, "Theory and Practice of Infrared Technology for Non-Destructive Testing", (New York: Wiley), 2001.

7. Vladimir P.Vavilov and Douglas D.Burleigh, "Review of pulsed thermal NDT: Physical principles, theory and data processing", NDT\&E International73 (2015)28-52.

8. PPT Ibarra-Castanedo C. and Maldague X. "Pulsed Phase Thermography Reviewed," Proceedings of QIRT 7 -Quantitative Infrared Thermography, 2004. Poster.

9. S. Pickering, D. Almond, "Matched excitation energy comparison of the pulse and lock-in thermography NDE techniques", NDT E Internat. 41 (7) (2008) 501-509.

10. Ravibabu Mulaveesala, Jyani Somayajulu Vaddi, and Pushpraj Singh, "Pulse compression approach to infrared nondestructive characterization", Review Of Scientific Instruments 79, 094901-2008.

11. V Arora, J A Siddiqui, R Mulaveesala and A Muniyappa, "Hilbert transform-based pulse compression approach to infrared thermal wave imaging for sub-surface defect detection in steel material", Insight Vol 56 No 10 October 2014 


\section{Numerical Simulation of Non-Destructive Characterization of CFRP Composite Through Frequency Modulated Thermal wave Imaging}

12. Suresh B., Subhani S., Vijayalakshmi A., Vardhan V.H., Ghali V.S., Chirp Z transform based enhanced frequency resolution for depth resolvable non stationary thermal wave imaging,2017 Review of Scientific Instruments, Vol:88, issue:1, DOI: 10.1063/1.4973192, ISSN: 346748.

13. Subhani S.K., Suresh B., Ghali V.S., Empirical mode decomposition approach for defect detection in non-stationary thermal wave imaging ,2016, NDT and E International, Vol: 81, Issue: pp: 39 - 45, ISSN 9638695.

14. Sivavaraprasad Gampala, D. Venkata Ratnam and Yuichi Otsuka, "Multicomponent Analysis of Ionospheric Scintillation Effects Using the Synchrosqueezing Technique for Monitoring and Mitigating their Impact on GNSS Signals", November 2018 Journal of Navigation 72(3):1-16

15. J.L Ravipati, A Vijayalakshmi,B.Chandana and V.S.Ghali," Multi Resolution based Enhanced Subsurface Analysis in thermal Wave Detection and Ranging," JARDCS,9, pp.2005-2013(2017).

16. V.S.Ghali, S.Sukumar, D.L.Srinivas, K.L.Mallika and Sk.Subani, "Principal component non stationary thermal wave imaging", International journal of systems and Technologies, Vol 6, Issue 2, 2013, PP 6-10.

17. Javed Ahmad, Aparna Akula, Ravibabu Mulaveesala, and H. K. Sardana, "Barker-Coded Thermal Wave Imaging for Non-Destructive Testing and Evaluation of Steel Material', IEEE Sensors Journal, Vol. 19, No. 2, January 15, 2019.

18. S.Subhani, B.Suresh and V.S.Ghali, "Orthonormal Projection approach for depth - resolvable subsurface analysis in non - stationary thermal wave imaging," Insight, 58(1), pp.42-45 (2016).

19. Deboshree Roy, Prabhu Babu, and Suneet Tuli, "Sparse Reconstruction-Based Thermal Imaging for Defect Detection”, IEEE Transactions On Instrumentation And Measurement, Volume: 68, Issue: 11, Nov. 2019

20. GV.Subbarao and J.L.Ravipati," An improved image denoising method using compressive sensing based thresholding method," JARDCS, 10,pp. 703-711(2018).

21. Anil Kumar D., Kishore P.V.V., Venkatram N., Leela Rani B., Hasitha A., Sabarish T.V.N.S.H., Sai Chandra R., Indian sign language recognition: A comparison between ANN and FIS ,2016, Journal of Theoretical and Applied Information Technology, Vol: 89, Issue: 2, pp: $502-511$.

22. Kishore P.V.V., Kumar D.A., Goutham E.N.D., Manikanta M. , Continuous sign language recognition from tracking and shape features using Fuzzy Inference Engine ,2016 , Proceedings of the 2016 IEEE International Conference on Wireless Communications, Signal Processing and Networking, Wisp NET 2016 ,Vol: ,Issue: ,pp: 2165 - 2170.

23. Vijaya Lakshmi A., Gopitilak V., Muzammil Parvez M., Subhani S.K., Ghali V.S., "Artificial neural networks based quantitative evaluation of subsurface anomalies in quadratic frequency modulated thermal wave imaging", Infrared Physics and Technology, ISSN: 13504495, Vol No:97, 2019, pp:108-115.

24. Vijaya Lakshmi, V. S. Ghali, Muzammil Parvez M, G V. P. Chandra Sekhar Yadav, V. Gopi Tilak, “ Fuzzy C-Means Clustering Based Anomalies Detection in Quadratic Frequency Modulated Thermal Wave Imaging”, International Journal of Recent Technology and Engineering (IJRTE), Volume-8 Issue-3, September 2019.

25. Suresh B., Subhani Sk., Ghali V.S., Mulaveesala R., Subsurface detail fusion for anomaly detection in non-stationary thermal wave imaging,2017 Insight: Non-Destructive Testing and Condition Monitoring, Vol:59, issue:10, pp: 553-558.

\section{AUTHORS PROFILE}

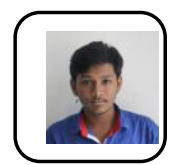

T. S. V. Saketh, pursuing his B. Tech from Infrared Imaging Center, Department of Electronics and Communication Engineering, Koneru Lakshmaiah Education Foundation, Vaddeswaram, AP, India.

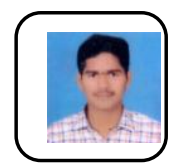

V. N. S. G. Sathwik, pursuing his B. Tech from Infrared Imaging Center, Department of Electronics and Communication Engineering, Koneru Lakshmaiah Education Foundation, Vaddeswaram, AP, India.

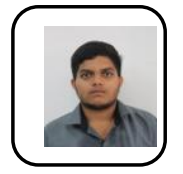

D. V. Sai Prahlad, pursuing his B. Tech from Infrared Imaging Center, Department of Electronics and Communication Engineering, Koneru Lakshmaiah Education Foundation, Vaddeswaram, AP, India.

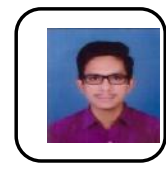

V. Gopi Tilak received his B. Tech degree in Electronics and communication engineering from JNTU Kakinada in 2016 and $M$. Tech in communication and radar systems from KL University in 2018 and his area of interest includes Infrared Thermography, Non Destructive Testing \&
Evaluation, Thermal wave Imaging and Machine learning. Presently he is pursuing his Ph. D from Infrared Imaging Center, Department of Electronics and Communication Engineering, Koneru Lakshmaiah Education Foundation, Vaddeswaram, AP, India.

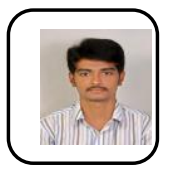

G.V.P Chandra Sekhar Yadav received his B. Tech degree in Electronics and Communication Engineering from JNTU Kakinada in 2012, M. Tech degree in Digital Electronics \& Communication Systems from JNTU Kakinada in 2015 and his area of interest includes Infrared Thermography, Non Destructive Testing \& Evaluation, Thermal wave Imaging and Signa processing. Presently he is working as a Research scholar at Infrared Imaging Center, Department of Electronics and Communication Engineering, Koneru Lakshmaiah Education Foundation, Vaddeswaram, AP, India.

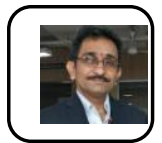

Dr. V. S. Ghali (M94255510) received his M. Sc degree in Electronics from Acharya Nagarjuna University, Guntur, India, in 1998 and the M.E degree in Applied Electronics from Satyabama University, Chennai, India in 2008. He received his Ph. D degree in ECE from IIITDM, Jabalpur India in 2013. He is a recipient of research award from University grant commission, India in 2014. Currently he belonged to Infrared Imaging Center and also Professor in Department of Electronics and Communication Engineering, Koneru Lakshmaiah Education Foundation, Vaddeswaram, AP, India. 